\title{
Arduino-controlled Reflectance Transformation Imaging to the study of cultural heritage objects
}

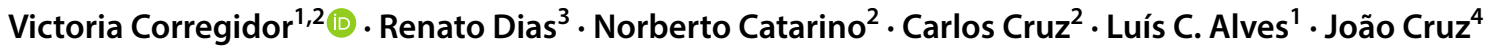

Received: 4 March 2020 / Accepted: 16 August 2020 / Published online: 29 August 2020

(c) Springer Nature Switzerland AG 2020

\begin{abstract}
This article examines the development of a low-cost and portable set-up controlled by an Arduino board to perform Reflectance Transformation Imaging technique, from the information derived from 45 digital photographs of an object acquired using a stationary camera. The set-up consists of 45 high-intensity light emitting diodes (LEDs) distributed over a hemispherical dome of $70 \mathrm{~cm}$ in diameter and a digital camera on the top of the dome. The LEDs are controlled by an Arduino board, and the user can individually control the LEDs state (ON or OFF) and duration of illumination. An old manuscript written with iron-gall ink and a set of 1 Euro coins mint in 2002 were photographed with the set-up. The interactive re-lighting and the mathematical enhancement of the object's surface revealed corrosion, loss of material, scratches and other details, which were not perceived in standard images. These unique features, which can be extracted using edge detection processing, have immediate application in different fields such as cultural heritage or forensic studies, where they can be used as fingerprints to identify unique objects, allowing also recognizing the use of tools to alter the surface of coins to increase the price in the market.
\end{abstract}

Keywords Arduino $\cdot$ Low-cost dome $\cdot$ Reflectance Transformation Imaging $\cdot$ Coins recognition $\cdot$ Degradation visualization

\section{Introduction}

Reflection Transformation Imaging (RTI) technique allows the visualization of the relief of the surface of an object allowing a better representation than the one obtained through a single standard image, since surface self-shadowing or inter-reflections are recorded, enhancing thus the realism of the final image.

RTI images offer several advantages when compared to standard images, providing a quick overview of the texture of an object and enhancing details of the image by a moving virtual light source. Results from RTI technique were very useful to identify morphological changes [1, 2], craquelure, planar distortion, wood grain, canvas weave and pentimenti on several paintings in a museum environment [3]. In this context, when compared with traditional raking light photography, $\mathrm{RTI}$ offers several advantages such as the control and knowledge of the exact position and angle of the light and thus the reproducibility of the results.

Electronic supplementary material The online version of this article (https://doi.org/10.1007/s42452-020-03343-4) contains supplementary material, which is available to authorized users.

$\triangle$ Victoria Corregidor, vicky.corregidor@ctn.tecnico.ulisboa.pt | ${ }^{1}$ Centro de Ciências e Tecnologias Nucleares, Instituto Superior Técnico, Universidade de Lisboa, E.N. 10 ao km 139,7, 2695-066 Bobadela LRS, Portugal. ${ }^{2}$ IPFN, Instituto de Plasmas e Fusão Nuclear, Instituto Superior Técnico, Universidade de Lisboa, 1049-001 Lisboa, Portugal. ${ }^{3} \mathrm{MEEC}$, Instituto Superior Técnico, Universidade de Lisboa, 1049-001 Lisboa, Portugal. ${ }^{4}$ Laboratório de Instrumentação, Engenharia Biomédica e Física da Radiação ((LIBPhys- UNL), Departamento de Física, Faculdade de Ciências e Tecnologia da, Universidade Nova de Lisboa, Monte da Caparica, 2892-516 Caparica, Portugal. 
The possibility to visualize surface details otherwise hidden in a standard photography can be one of the reasons why this technique is being widely used to study cultural heritage objects, led by the US nonprofit corporation "Cultural Heritage Imaging" [4]. This corporation, jointly with a team from the Universidade do Minho (Portugal) [5], were the primary developers of the RTI Builder tool [6]. It enables to manage all aspects of building Reflectance Transformation Images, also known as rti files using the Polynomial Texture Mapping (PTM) fitter software, developed by HP [7], which allow to compute an approximation of the reflectance function at each pixel of an image obtained using a fixed digital camera and a moveable, stable light source in a way that the distance between the light source and the surface remains constant.

RTI technique allows the visualization of the surface details which are enhanced by the different light directions and the computation, at each pixel of the image, of the reflectance function. For this, it is assumed that the reflectance varies with the light direction while the chromaticity is independent of it. The use of the PTM fitter software requires the knowledge of the light source position for each digital image as input data, to reconstruct the reflectance function. This can be easily computed from the coordinates of the geometric centre of a glossy dark sphere (which is next to the object under investigation, remaining fixed across the image set acquisition, and also photographed) and the geometric centre of each highlight reflection in the sphere [8].

The RTI Builder software detects the glossy dark sphere in the input digital images and produces the light vector positions required to build the rti file. Additionally, the RTI Viewer software [9] or WebRTIViewer [10] enables to view and explore those $r t i$ files, allowing the user interaction as the possibility to move light to any angle, or to apply different mathematical filters available, such as diffuse gain, unsharp image or normal visualization.

RTI technique can use low-cost and non-specialized equipment, being the camera and lens the most expensive components. It is also relatively easy to perform, but some guidelines should be considered such as the perfect control of light intensity constancy during the shots (constant illumination from each direction), and the stability of the camera, object and glossy dark sphere during the capture sequence.

Different set-ups have been developed to obtain RTI images. Earl et al. [11, 12] employed two types of RTI systems: a monitored rotating arc which can have up to sixteen low-output LED light sources and a fixed camera; a hemispherical mesh where light sources are placed and a fixed camera, used for small objects. Newman et al. reproduced the surface of bones using a moveable light source (handheld flash) around the object along the surface of a "virtual light dome" [13] defined at a fixed distance from the object. Porter et al. [14] developed a portable rig out of PVC sheets consisting of a rotating arm with small flashlight fixed at different heights and angles, and a circular ring to guide the placement of the arm at fixed intervals around the object. Morita et al. [15] developed a RTI system where illumination was made by a 168 LED lamp manually operated for small objects or by an halogen lamp for big objects (a column of a cemetery). Piquette [16] developed a dome of $1 \mathrm{~m}$ in diameter with 76 LED light sources located at different positions around each circle at $15^{\circ}, 40^{\circ}$, and $65^{\circ}$. Other dome design can be found in [17], where Palma et al. developed a dome of aluminium shells with 116 cold white LEDs, computer controlled synchronizing the switching on of each LED with the shutter of the camera.

Examples of RTI applications in the field of cultural heritage are diverse [11-20]. In general, small objects such as bone artefacts $[13,15]$ or metallic objects (mostly coins) [12,17, 19-21] are the most characterized. With RTI it is possible to enhance the visibility of intentional modifications, tool marks, engraving details, etc. $[7,11,14,16,19]$ being very useful to document the objects. In the case of archaeological textiles, the examination is usually made by visual inspection with low power magnification, but the use of micro-RTI (by using a microscopy) allowed the visual representation of the weave, fibres, surface and patterns of decay and erosion [22], avoiding inadequate handling and misidentification of raw materials. Regarding ancient documents (parchment, papyrus or paper media written with irongall inks or other pigments), RTI technique can be used to digitalize them, allowing the visualization to external researchers without the need of handling. Furthermore, combining RTI with spectral imaging [23, 24], also known as spectral RTI, it can be possible to read illegible letters, distinguish letters or symbols, and visualize the evidence of corrosion, or ink stains that cannot be distinguished by the human eye.

In this work are shown results from two different objects (an old manuscript and coins) acquired with a setup developed by the group. The system was conceived for allowing it to be used in several different locations to study a wide variety of objects (size and materials), and being easily operated by anyone, then combining simplicity, versatility and portability with the use of low-cost (and lightweight) materials. Considering the set-up developed by other researchers $[3,11,12,14-17,23]$, a hemisphere dome with 45 high-intensity LEDs made by aluminium and 3D printed pieces was developed. The use of an Arduino board allows controlling the LEDs and the camera and thanks to a user-friendly GUI interface it is possible to control the set-up and take pictures easily. 
The objects chosen in this work show the versatility of the set-up, which is capable of analysing small and large objects (about $30 \mathrm{~cm} \times 30 \mathrm{~cm}$ ). In the case of the manuscript, this set-up arises with the need of searching nondestructive techniques to analyse documents with irongall ink which suffers from foxing stains and degradation due to the ink and inappropriate storage conditions and manipulation. By using this technique as a first approach in the characterization, regions of interest can be easily found to be further studied by other non-destructive techniques [25-27]. In fact, due to the reproducibility of the RTI technique, it can be used as a method to study the progress of degradation over time and thus be able to take appropriate conservation and restoration procedures [24].
In the case of coins, it is intended to go a step further in the characterization of this type of object. The objective is to check whether the coins can be individually characterized by the surface irregularities. They may appear during the minting process (e.g. a crack in the die leaves a distinct mark on the coin surface) and/or afterwards on the daily circulation. They range from almost unnoticed to very pronounced and its pattern, enhanced by mathematical algorithms [28], can provide a unique fingerprint of the coins. This is appealing in the market of expensive/rare coins by providing a unique identification of each coin in case of loss or theft. Also, forgers' marks of surface manipulation can be disclosed, being a very useful technique for the forensic science. a

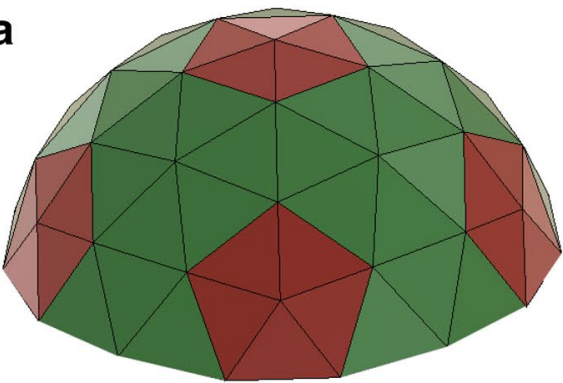

b

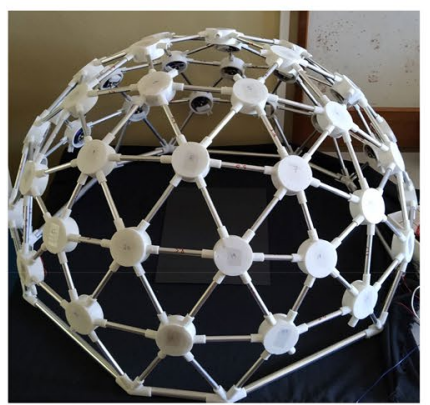

C
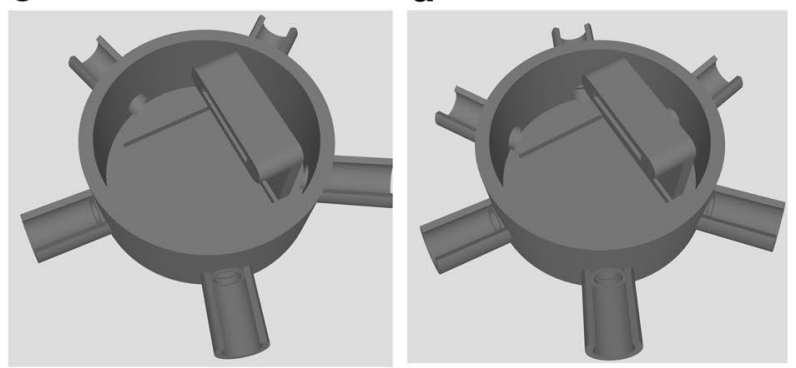

Fig. 1 a Representation of a 3 V $3 / 8$ using the Fusion 360 software; b the final $3 \mathrm{~V} 3 / 8$ dome set-up constructed with LEDs in the vertices fit in the plastic junctions designed using Fusion 360 software and printed with a 3D printer using PLA filament; c junction for lengths type AAAAA; $\mathbf{d}$ junction for lengths type CCCCCC; e junc- e

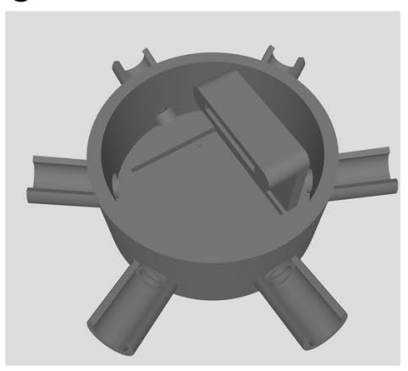

f

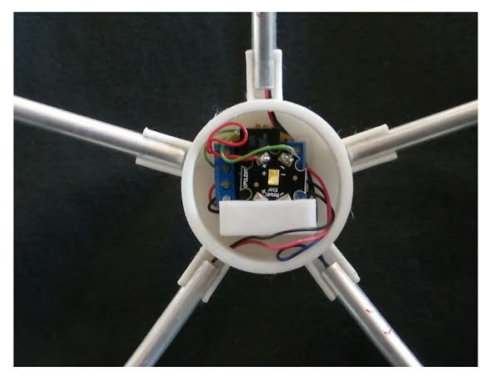

tions for lengths $A B B B C C$ (Lengths $A, B$ and $C$ are referred in the text); $\mathbf{f}$ photograph of a plastic junction where tubes fit by a cylindrical section opened $116^{\circ}$ and detail of how driver and LED group are positioned in the junction 
In both cases, and in general, although there are available other analytical techniques such as optical microscopy (2D or 3D), 3D-scanners to visualize in detail the surface of cultural heritage objects or the measurement of the roughness and reflectance spectra (colour) of the surface of an object to be used as a fingerprint of it [29], RTI technique offers the extra possibility to external researchers to investigate the objects by obtaining the corresponding $r$ ti file of each object, avoiding extra manipulation or transportation or even surface preparation. Furthermore, digital photographs can be taken in the object's environment, since the set-up is portable, preserving thus the object state.

\section{Experimental set-up and materials}

\subsection{System set-up}

The set-up used to capture the images of the objects is composed of 45 high-intensity light emitting diodes (LEDs), an Arduino MEGA board and a digital Canon camera. The LEDs are distributed over a hemisphere which allows the use of the PTM fitter and also a even distribution of the light directions at a constant distance from the image centre.

The hemisphere is a 3 V $3 / 8$ Geodesic Dome with 45 vertices and $0.70 \mathrm{~m}$ in diameter. The edges are made of aluminium hollow tubes with two dimensions: $8 \mathrm{~mm}$ in diameter for the edges and $12 \mathrm{~mm}$ in diameter for the base. These dimensions were chosen to accommodate the connection cables, being the base bigger to better support the dome structure. Also, there are three types of edges with different lengths: A:6.60 cm, B: $8.52 \mathrm{~cm}$ and $C: 8.83 \mathrm{~cm}$, depending on their location in the dome: pentagon radius, edge of the pentagon or hexagon, and hexagon radius, respectively. The edges are joined by plastic junctions or hubs of $5 \mathrm{~cm}$ in diameter, designed with Fusion 360 software [30] and printed with a 3D printer (FlashForge Dreamer) using white PLA filament (see Fig. 1). There are also three types of junctions (also shown in Fig. 1) which depend on their position in the dome: joining lengths type AAAAA (Fig. 1c), joining lengths type CCCCCC (Fig. 1d) and joining lengths ABBBCC (Fig. 1e). The tubes fit into the hubs by a cylindrical section with an $116^{\circ}$ opening (see Fig. 1f). Furthermore, junctions of the base were also designed and printed, according to the design.

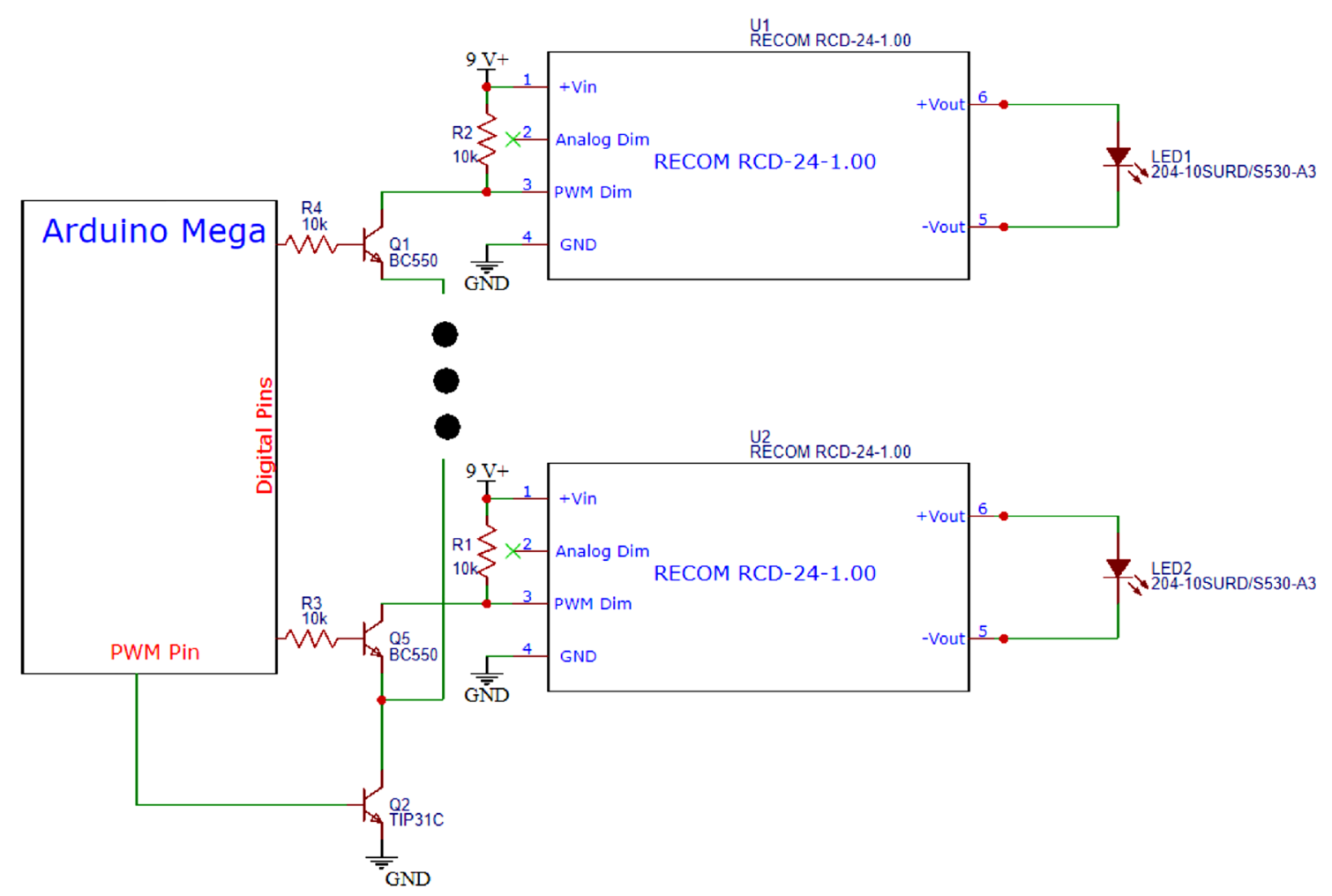

Fig. 2 Schematics for Arduino Mega in RTI system. Only two LEDs connected to the corresponding RECOM driver are shown. The three black dots represent the other 43 LED-driver groups 
Independent OPULENT Rebel-Start LEDs (LXW8-PW50) were used, which provide a high flux density and ANSI white light, similar to a flashlight. An advantage of using LEDs for illumination, and being ON during short time, is that limits the amount of damaging light on sensible objects, such as paper or paintings, and the thermal exposure is minimal. LEDs are distributed over the dome (they cover a range of inclination from 15 degrees above the horizon to 65 degrees above the horizon), jointly with a LED driver (RECOM RCD-24-1.00) with a current limit of 1 A, providing thus a constant-current output for LEDs and then constant flux illumination. Both, the driver and LED group, fit in the plastic junctions as it is shown in Fig. $1 \mathrm{f}$.

Prior to assemble the dome, each LED and driver group was tested. The test consists of measuring the luminous flux at $1 \mathrm{~m}$ from the light source by means of a ALX-1300 Lux Light Meter, discarding those groups with lower or higher flux. Each LED-driver group used for the set-up have a similar illuminance (110 Ix).

An Arduino MEGA 2560 microcontroller board based on the ATmega2560 is used to individually control each LEDdriver group. It has 54 digital input/output pins (of which 15 can be used as PWM, pulse width modulation, outputs) and 16 analog inputs [31]. The use of Arduino boards to develop acquisition systems in different fields of science is increasing along the years, see for example [32, 33].

A scheme of the circuit is shown in Fig. 2. For the LEDs status to be OFF by default, when turned on the set-up, the PWM value of the control signal must be $100 \%$, so a pull-up resistance $(10 \mathrm{k} \Omega$ ) is included (R1 and R2 in Fig. 2). It should be noted that all connections and cables are not visible, favouring the aesthetics of the set-up. Furthermore, the system is designed in such a way that it can be divided in half to facilitate transportation.

The images are captured by means of a Canon EOS $700 \mathrm{D}$ camera placed on the top of the dome in a stable and horizontal position (checked with a bubble level), being the camera-object distance of $37 \mathrm{~cm}$. In this configuration, the camera views the object from directly above. To avoid movements or vibrations, the camera can be remotely controlled, either using remote capture software or by the Arduino board. The camera and the Arduino board can be connected to a portable PC, used also to process the $r$ ti files after acquisition. Size of images acquired is $5184 \times 3456$ pixels.

Digital photographs of the document were acquired using a focal length of $35 \mathrm{~mm}$, with $\mathrm{f} / 6.3$ and exposure $1 / 10 \mathrm{~s}$, fixed for all the acquisitions. Digital photographs of the 1 Euro coins were acquired using a focal length of $135 \mathrm{~mm}$. They were taken with $\mathrm{f} / 6.3$ and exposure $1 / 4 \mathrm{~s}$, fixed for all the acquisitions.
The glossy dark sphere to compute the light direction is positioned near the object, using a matte black fabric as background to avoid unwanted reflections and shadows.

Illumination and acquisition can be controlled either from the computer by means of a dedicated developed GUI interface, where it is possible to control the trigger mode (automatic or manual) and trigger time, or from an external unit where it is located the Arduino board, the needed circuits and a LCD screen where it is also possible to choose the trigger mode and trigger time for illumination.

After acquisition, the images are imported to the RTI Builder software to create the rti file to be read by the RTI Viewer software. The later allows to virtually illuminate from any direction and to apply different rendering modes that enhance the details of the object's surface. More details are present in [6].

\subsection{Objects}

\subsubsection{Manuscript}

The manuscript studied, from a private collector, is a document from the XVIII century, written on paper with irongall ink. It is a collection of the Laws published during the reign of D. Maria I of Portugal (1734-1816, queen since 1777); it has 8 pages with dimensions of $20 \mathrm{~cm}$ width and $29 \mathrm{~cm}$ height. It suffers from loss of material, fox stains and degradation due to the ink acidity and poor storage conditions and manipulation.

\subsubsection{Coins}

The coin collection consists of $10 € 1(7.50 \mathrm{~g}$ and $23.25 \mathrm{~mm}$ in diameter) mint in 2002 by the Portuguese Mint House [34]. The $€ 1$ golden outer part is made of nickel brass, and the silver inner part has three layers: copper-nickel, nickel, copper-nickel [35]. The euro coins have one side which is common to all EU countries (reverse), and the other side (obverse) is specific for each country. For Portugal, the centrepiece is the royal seal of 1149 from D. Afonso I, the first Portuguese king (1109-1185, king since 1139). The introduction of Euro banknotes and coins in 2002 was a large currency changeover, near 52 billion coins were produced by the 12 participating countries, and 100.228 .135 pieces of $€ 1$ coins were mint in Portugal.

\subsection{Processing methods}

The scratches pattern on coins was extracted using image processing methods integrated in the Wolfram Mathematica computing system [36]. The edge detection function 


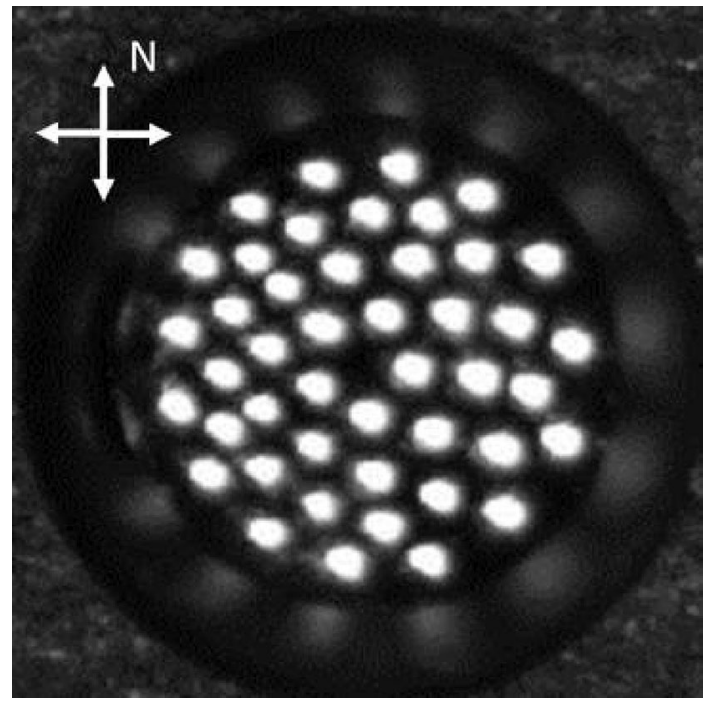

Fig. 3 Blended image showing all the highlights in the set of photographs (45) used to create the rti file

(finds edges in image and returns the result as a binary image) was applied to each input image, adjusting for each one the scale of the specified pixel range ( $r$ in the function) and the threshold $(t)$ to enhance the main features. Then, Color Negate function was applied to obtain the negative image, where it is easier to visualize the features found.

The input images are those images exported from RTI Viewer after application of a specific filter. After several attempts, the Specular Enhancement rendering mode, was found to be the most appropriate since the scratches can be easily enhanced and detected.

\section{Results and discussion}

\subsection{RTI set-up}

As has been previously stated above, the success of RTI technique relies on the knowledge of light source direction. The illumination of each LED should be invariable and positioned at a constant distance from the image centre. Figure 3 is a blended image showing all the highlights in the set of 45 photographs used to create the $r t i$ file for each object studied. This image is created by the RTI Builder software, which detects the highlights on the sphere and computes the light positions. As can be observed the LEDs are eventually distributed at the dome which is very important for the quality of the $r$ ti file as can be revealed when using the RTI Viewer software where almost all virtual light incidence directions can be

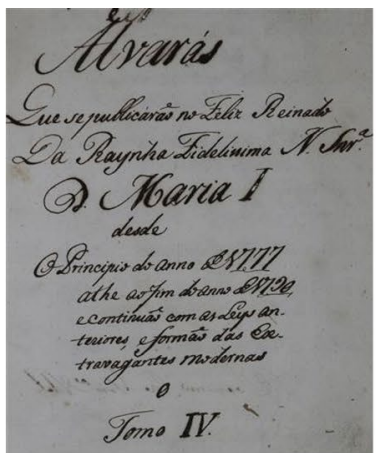

$\mathbf{a}$

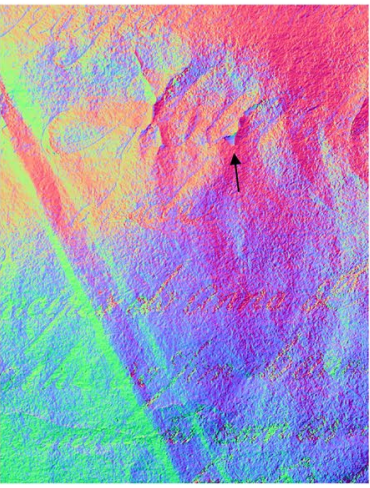

c

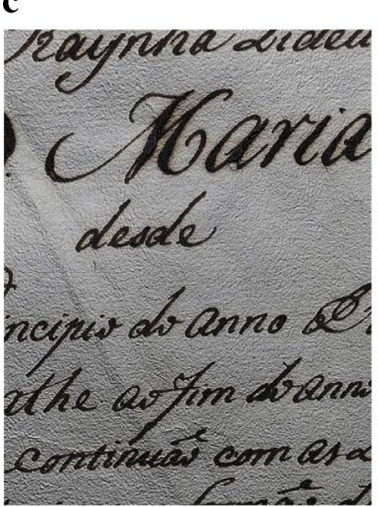

e
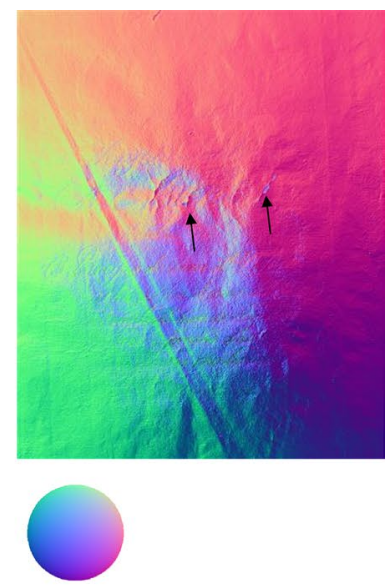

b

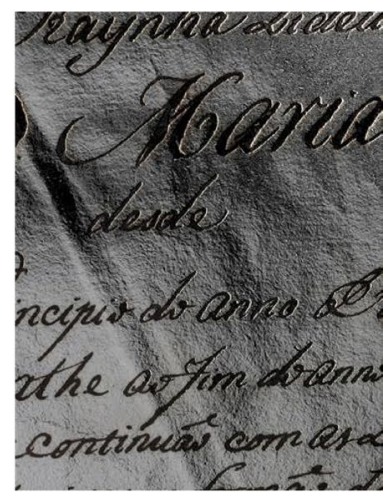

d
Fig. 4 Different displays of a rti file that belongs to the cover page of the collection of Laws published during the reign of Queen D. Maria I (XVIII century). a Default visualization; b normals visualization viewing mode; $c$ details of image with normals visualization viewing mode, showing a missing part of the paper; $\mathbf{d}$ details of image with Diffuse Gain rendering mode; e same detail but using Luminance Unsharp Masking rendering mode

reproduced, specially for some rendering modes such as multi-light enhancement modes, which choose different lighting angles for parts of an image in order to enhance surface details, optimizing sharpness and brightness. 
Different filters available in the RTI Viewer were applied, depending on the object studied, in order to extract as much information as possible.

\subsection{Manuscript}

Figure 4a shows the default image (with illumination from the top of the dome), without any filter of the cover page of the document under study. Some stains, foxing and some white parts, for example in "a" and "I" from D. Maria I, can be observed.

Deformation, wrinkles and folds are more evident when using the normals visualization viewing mode of the same image (see Fig. 4b). This viewing mode creates a false color image considering the surface normal direction information at each pixel of the image [21]. Using this mode, it is easier to observe that the white parts observed in Fig. 4a are in fact missing parts of the support (paper) as can be seen in more detail in Fig. 4c. They are probably related to ink acidity and endogenous factors, such as the acid hydrolysis of cellulose $[25,26]$.

Figure $4 d$, e corresponds to a detail of the cover page, using Diffuse Gain rendering mode with raking light from the north side and Luminance Unsharp Masking rendering mode with raking light from the east side of the dome, respectively. The Diffuse Gain rendering mode enhances the perception of surface shape of the objects, since it represents the directional rate of change of surface normal direction, by computing the second derivative. In this way, not only the wrinkles are more evident but also the smooth texture of letters written with iron-gall ink. On the other hand, the Luminance Unsharp Masking rendering mode enhances the highfrequency details, increasing the depth discontinuities (edge contrast) without affecting the colour. Then, in Fig. $4 \mathrm{~d}$ texture of paper is enhanced and ink diffusion in some letters (for example in " $\mathrm{M}$ " or in the boundaries of both " $a$ " for Maria) becomes more evident than in the default image. These features can be considered in \#coin Specular Enhancement

(A)

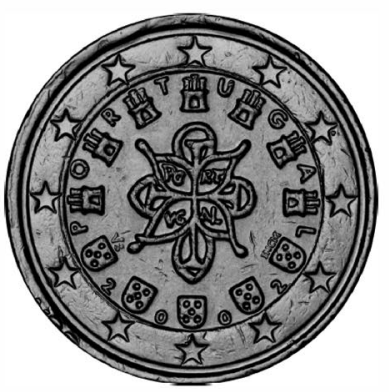

2
Normal Unsharp

Masking

(B)
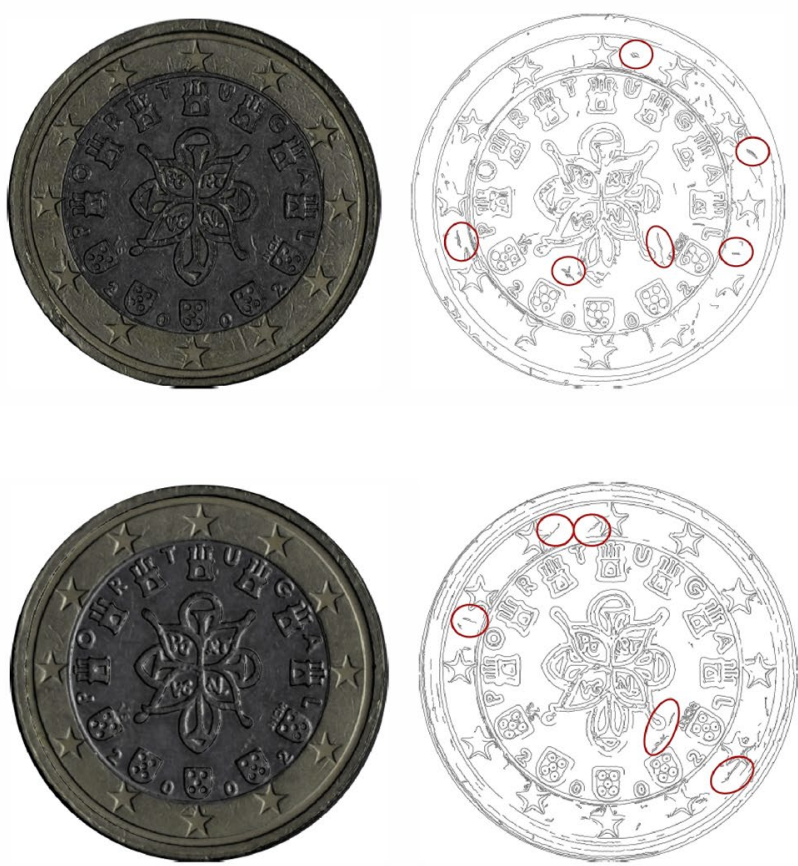

Edge Detection

(C)

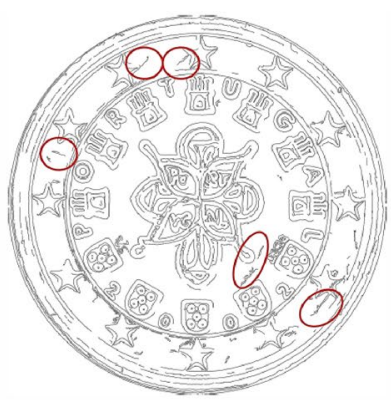

1

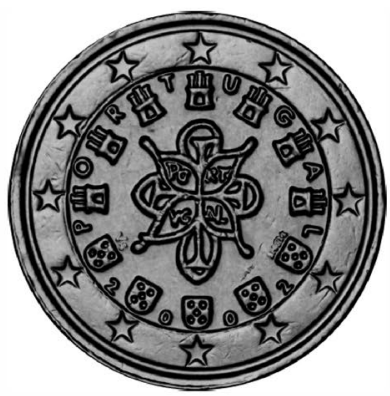

Fig. 5 RTI images using: a specular enhancement rendering mode; $\mathbf{b}$ normal unsharp masking rendering mode; $\mathbf{c}$ edge detection and features 
future studies of this document involving non-destructive techniques to infer the origin, which can be related with the presence of impurities in the iron-gall ink such as $\mathrm{Zn}$ or $\mathrm{Cu}$ ions and Fenton reactions [37]. Furthermore, these features can be monitored by the RTI technique to study the evolution of the deterioration and degradation during storage or to control the evolution after conservation treatments [24].

\subsection{Coins}

When coins or metallic objects are photographed, unwanted specular reflections and shininess usually appear in the images. Specular Enhancement rendering mode removes the diffuse RGB colour of the digital photographs and also the specular reflections and the specular highlights, resulting thus in the enhancement of the surface shape, making it easier to focus the attention on fine surface details, such as engravings and scratches of the coins, as shown in Fig. 5.

Furthermore, Unsharp Masking rendering mode can be also a useful tool to enhance engraved areas. As described above, this mode enhances the high-frequency details, increasing the edge contrast, using the surface normals vectors, as shown the same coins with this rendering mode in Fig. 5.

In both cases surface morphology and individual engravings are more recognizable than in a traditional digital photograph. Furthermore, by using Normal Unsharp rendering mode, new details appeared, increasing the visibility of the edges of the designs.

The signs of usage of these coins ( 17 years old) can be imaged and used as fingerprint for each coin, then allowing their identification among similar coins, or as a proof of property in case of theft.

The scratches pattern for each coin extracted using image processing methods is shown in Fig. 5 and supplementary information. The red circles highlight the features found for each one of them, which are extracted by individually adjusting the $r$ and $t$ parameters in the edge detection function. From these figures it is clear that each coin has a unique set of features that can be used to identify without doubt each coin among the set. Most of the marks are located in the highest part of the coins, as expected, related to the usage of coins in daily life.

This example can be extrapolated to collector coins, which can be forged by experts, but probably they cannot reproduce the exact pattern of scratches of each coin.

RTI technique can be used as the first technique to characterize the objects, for instance coins with doubtful authenticity, and then proceed to other scientific methods to check if the composition matches with other coeval coins or objects, see for example [38-41].

\section{Conclusions}

A versatile, portable and automatized RTI set-up has been developed. The system, based on an Arduino MEGA 2560 board was found to be suitable for studying different types of cultural heritage objects. The low cost and ease of use of the system makes it suitable for a wide range of applications in educational and research environment.

Surface modifications in documents are enhanced by RTI technique, offering evidence of iron-gall ink migration, surface topography, the texture of paper and ink details not shown in standard photographs. The use of Normal visualization viewing mode is a very quick way to check the flatness and also the loss of material support in certain places. In this way the RTI can be used to determine the areas which need a conservation or restoration strategy. Furthermore, taking sets of digital photographs of the document under the same light conditions along the time can allow determining the evolution of these areas by monitoring surface normals, due to endogenous or exogenous factors.

Visible alterations represented when using RTI technique to coins surfaces can reveal their history on account of production techniques marks and usage patterns. These alterations can be used as fingerprints to identify coins, allowing also to recognize tools application to alter the surface of coins in order to increase their price in the market.

Edge detection function has been applied individually for each coin, but the processing method should be extended to an automatic processing, testing other functions to detect regions of interest not only on 1 Euro coins.

Images acquired allow a better documentation of the objects, and they can be analysed by remote researchers, interested stakeholders or the general public, avoiding then the transportation of valuable or fragile objects, and related costs as insurance. Likewise, the manipulation of these objects is minimized, ensuring their conservation state by reducing the external factors that can affect it.

Acknowledgements Support from C.Chaves, L.M. Ferreira, M. Fonseca, J. Rocha, E. Alves, T. Fonseca, C. Silva, F. Azevedo is acknowledged. Special thanks to R. Winstanley for sharing with us his collection. C. Chu and Impersol Lda. is acknowledged for providing pieces for the set-up. Special thanks to Ciência Viva participants for their help, previous students (C. Silva from Universidade Nova de Lisboa and F. Azevedo from Instituto Superior Técnico) for their involvement during the RTI set-up design. 
Funding Funding was provided by Fundação para a Ciência e a Tecnologia, Portugal (Grant Nos. UIDB/ 04349/2020 and UID/ FIS/04559/2019) and private funds. V.C. acknowledges the support from UID/Multi/04349/2019. J.C. acknowledges NOVA.ID.FCT.

\section{Compliance with ethical standards}

Conflict of interest The authors declare that they have no competing interests.

\section{References}

1. Manfredi M, Bearman G, Williamson G, Kronkright D, Doehne $E$, Jacobs M, Marengo E (2014) A new quantitative method for the non-invasive documentation of morphological damage in paintings using RTI surface normals. Sensors 14:12271-12284

2. Tamayo SNM, Andres JV, Pons JO (2013) Transformation imaging for documentation and surface analysis in conservation. J Conserv Sci 4:535-548

3. Padfield J, Saunders D, Malzbender T (2005) Polynomial texture mapping: a new tool for examining the surface of paintings, ICOM committee for conservation. In: Proceedings of the 14th triennial meeting. pp 504-510. https://cima.ng-london.org.uk/ ptm/index.html. Accessed 19 July 2020

4. $\mathrm{CHI}$, Cultural Heritage Imaging, in. http://culturalheritageimag ing.org/Technologies/RTI/. Accessed 19 July 2020

5. Barbosa JG, Sobral JL, Proença AJ (2007) Imaging techniques to simplify the PTM generation of a bas-relief. In: Proceedings of the the 8th international symposium on virtual reality, archaeology and cultural heritage. pp 28-31

6. RTI_Builder, Cultural Heritage Imaging, in. http://culturalheritag eimaging.org/What_We_Offer/Downloads/Process/index.html. Accessed 19 July 2020

7. Malzbender T, Gelb D, Wolters H (2001) Polynomial texture maps. In: Proceedings of the 28th annual conference on computer graphics and interactive techniques. pp 519-528. https:// doi.org/10.1016/j.jas.20

8. Mudge M, Malzbender T, Schroer C, Lum M (2006) New reflection transformation imaging methods for rock art and multiple-viewpoint display. In: Proceedings of the 7th international conference on virtual reality, archaeology and intelligent cultural heritage. pp 195-202. https://doi.org/10.2312/vast/vast0 6/195-202

9. RTI_Viewer, Cultural Heritage Imaging, in. http://culturalheritag eimaging.org/What_We_Offer/Downloads/Process/index.html. Accessed 19 July 2020

10. WebRTIViewer, WebRTIViewer, in. http://vcg.isti.cnr.it/rti/webvi ewer.php. Accessed 19 July 2020

11. Earl G, Martinez K, Malzbender T (2010) Archaeological applications of polynomial texture mapping: analysis, conservation and representation. J Archaeol Sci 37:2040-2050. https://doi. org/10.1016/j.jas.2010.03.009

12. Earl G, Basford PJ, Bischoff AS, Bowman A, Crowther C, Dahl J, Hodgson M, Martinez K, Isaksen L, Pagi H, Piquette KE, Kotoula E (2011) Reflectance Transformation Imaging systems for ancient documentary artefacts. In: EVA London 2011: Electronic Visualisation and the Arts. pp 147-154. https://eprints.soton.ac. uk/204531/. Accessed 19 July 2020

13. Newman SE (2015) Applications of Reflectance Transformation Imaging (RTI) to the study of bone surface modifications. J Archaeol Sci 53:536-549
14. Porter ST, Huber N, Hoyer C, Floss H (2016) Portable and low-cost solutions to the imaging of Paleolithic art objects: a comparison of photogrammetry and Reflectance Transformation Imaging. J Archaeol Sci Rep 10:859-863

15. Morita MM, Novoa FD, Bilmes GM (2019) Reflectance Transformation Imaging. First applications in cultural heritage in Argentina. J Archaeol Sci Rep 26:101899

16. Piquette KE (2011) Reflectance Transformation Imaging (RTI) and ancient Egyptian material culture. Damqatum-The CEHAO Newsletter 7, 16-20. https://repositorio.uca.edu.ar/handl e/123456789/7350. Accessed 19 July 2020

17. Palma G, Badassari M, Chiara Favilla M, Scopigno R (2014) Storytelling of a coin collection by means of RTI images: the case of the Simoneschi collection in Palazzo Blu. In: Proctor N, Cherry R (eds) Museums and the web 2014. Silver Spring. http://vcg.isti. cnr.it/Publications/2014/PBFS14

18. Reedy CL (2018) Reflectance Transformation Imaging (RTI) in conservation. In: The encyclopedia of archaeological sciences. pp 1-6

19. Mytum H, Peterson JR (2018) The application of Reflectance Transformation Imaging (RTI) in historical archaeology. Hist Archaeol 52:489-503. https://doi.org/10.1007/s4163 6-018-0107-x

20. Hess M, MacDonald LW, Valach J (2018) Application of multimodal $2 \mathrm{D}$ and $3 \mathrm{D}$ imaging and analytical techniques to document and examine coins on the example of two Roman silver denarii. Herit Sci 6:5. https://doi.org/10.1186/s40494-018-0169-2

21. MacDonald LW (2014) Colour and directionality in surface reflectance. In: Proceedings of conference on artificial intelligence and the simulation of behaviour (AISB)

22. Goldman Y, Linn R, Shamir O, Weinstein-Evron M (2018) MicroRTI as a novel technology for the investigation and documentation of archaeological textiles. J Archaeol Sci Rep 19:1-10. https ://doi.org/10.1016/j.jasrep.2018.02.013

23. Vandermeulen B, Hameeuw H, Watteeuw L, Van Gool L, Proesmans M (2018) Bridging multi-light \& multi-spectral images to study, preserve and disseminate archival documents. Archiving 2018:64-69. https://doi.org/10.2352/issn.2168-3204.2018.1.0.15

24. Hanneken TR (2016) New technology for imaging unreadable manuscripts and other artifacts: integrated spectral reflectance transformation imaging (spectral RTI. In: Clivaz C, Dilley $P$, Hamidović D (eds) Ancient worlds in a digital culture. Digital Biblical Studies, Leiden, pp 180-195

25. Corregidor V, Viegas R, Ferreira LM, Alves LC (2019) Study of iron gall inks, ingredients and paper composition using nondestructive techniques. Heritage 2:2691-2703

26. Rouchon $V$, Duranton $M$, Burgaud $C$, Pellizzi E, Lavédrine $B$, Janssens K, de Nolf W, Nuyts G, Vanmeert F, Hellemans K (2011) Room-temperature study of iron gall ink impregnated paper degradation under various oxygen and humidity conditions: time-dependent monitoring by viscosity and X-ray absorption near-edge spectrometry measurements. Anal Chem 83:2589-2597

27. Viegas R, Corregidor V, Peña T, Alves E, Alves LC (2013) Preliminary studies on iron gall inks composition using an external ion beam. Int J Conserv Sci 4:593-602. www.ijcs.uaic.ro

28. Brognara C, Corsini M, Dellepiane M, Giachetti A (2013) Edge detection on polynomial texture maps, image analysis and processing-ICIAP 2013. pp 482-491

29. Wei W, Bakker V, Goodall JFS, Lagana A, Martinez K, Sotiropolou S, Walther A, Wiora G, Weber M (2008) A semi-automatic system for the non-contact 'fingerprinting' of objects of art and cultural heritage. In: Proceedings of the ICOM-CC 15th triennial meeting. pp 376-380

30. Autodesk, Fusion360, in. https://autodesk.com/products/fusio n-360/overview. Accessed 19 July 2020 
31. Arduino, Mega, in. https://store.arduino.cc/arduino-mega-2560rev3. Accessed 19 July 2020

32. Al-Obaidy F, Yazdani F, Mohammadi FA (2017) Intelligent testing for Arduino UNO based on thermal image. Comput Electr Eng 58:88-100. https://doi.org/10.1016/j.compeleceng.2017.01.014

33. Teikari P, Najjar RP, Malkki H, Knoblauch K, Dumortier D, Gronfier C, Cooper HM (2012) An inexpensive Arduino-based LED stimulator system for vision research. J Neurosci Methods 211:227236. https://doi.org/10.1016/j.jneumeth.2012.09.012

34. INCM, Imprensa Nacional Casa da Moeda, in. https://incm.pt/ portal/mpm_euro.jsp?lang=en. Accessed 19 July 2020

35. ECB, European Central Bank, in. https://ecb.europa.eu/euro/ coins/common/html/index.en.html. Accessed 19 July 2020

36. I. Wolfram Research, Mathematica, Version 12, in, Wolfram Research, Inc., 2020. https://www.wolfram.com/mathematica. Accessed 19 July 2020

37. Strlic M, Kolar J, Selih V-S, Kocar D, Pihlar B (2003) A comparative study of several transition metals in Fenton-like reaction systems at circum-netural PH. Acta Chim Slov 50:619-632
38. Borges R, Alves L, Silva RJC, Araújo MF, Candeias A, Corregidor V, Valério $P$, Barrulas $P(2017)$ Investigation of surface silver enrichment in ancient high silver alloys by PIXE, EDXRF, LA-ICP-MS and SEM-EDS. Microchem J 131:103-111

39. Borges R, Silva RJC, Alves LC, Araújo MF, Candeias A, Corregidor V, Vieira J (2018) European silver sources from the 15th to the 17th century: the influx of new world silver in Portuguese currency. Heritage 1:453-467

40. Cruz J, Corregidor V, Alves LC (2017) Simultaneous use and selfconsistent analyses of PIXE and EBS for the characterization of corrosion layers grown on ancient coins. Nucl Instrum Methods Phys Res Sect B 406:324-328

41. Pitarch A, Queralt I, Alvarez-Perez A (2011) Analysis of Catalonian silver coins from the Spanish War of Independence period (1808-1814) by Energy Dispersive X-ray Fluorescence. Nucl Instrum Methods Phys Res Sect B 269:308-312

Publisher's Note Springer Nature remains neutral with regard to jurisdictional claims in published maps and institutional affiliations. 